\title{
Défict de estatura para a idade de escolares do baixo Amazonas
}

\author{
Deficit of status for the age of low Amazon schools
}

\author{
Marcelo Gonçalves Duarte ${ }^{1}$ \\ Sueyla Ferreira da Silva dos Santos ${ }^{1}$ \\ Adriano Tavares de Carvalho ${ }^{1}$ \\ Giseli Minatto ${ }^{2}$ \\ Thais Reis Silva de Paulo ${ }^{1}$ \\ Glauber Carbalho Nobre $3,4^{*}$ \\ Cleene Tavares de Souza ${ }^{5}$ \\ Ismael Freitas Junior ${ }^{6}$
}

\section{Resumo}

Objetivo: investigar possível défict de estatura para idade de escolares (3 a 19 anos) de ambos os sexos de Parintins, AM. Métodos: pesquisa epidemiológica com delineamento transversal, em escolas públicas credenciadas ao Programa Saúde na Escola (PSE). O estado nutricional foi avaliado pelos indicadores de massa corporal, estatura e IMC e a adequação segundo as curvas de crescimento da Organização Mundial da Saúde. Resultados: observou-se predominância de baixa estatura, diferindo as proporções entre os meninos $(16,2 \%)$ e as meninas $(83,9 \%)$, em todas as faixas etárias. Conclusão: 0 déficit de estatura nas meninas, alerta para a necessidade de medida preventiva à desnutrição e déficit de crescimento.

Palavras-Chave: antropometria, crescimento corporal, saúde da criança, saúde do adolescente, estatura-idade.

\section{Abstract}

Objective: to investigate possible height-for-age deficit of schoolchildren (3 to 19 years old) of both genders of Parintins, AM. Methods: epidemiological research with a cross-sectional design, in public schools accredited to the Health in School Program (PSE). The nutritional status was evaluated by the indicators of body mass, height and BMI and the adequacy according to the growth curves of the World Health Organization. Results: a predominance of short stature was observed, differing the proportions between boys (16.2\%) and girls (83.9\%), in all age groups. Conclusion: The short stature in girls warns of the need for a preventive measure to malnutrition and growth deficit.

Key-words: anthropometry, body growth, child health, adolescent health, height-age.

Afiliação dos autores

${ }^{1}$ Universidade Federal do Amazonas, Parintins, Amazonas, Brasil.

'Universidade Federal de Santa

Catarina, Florianópolis, Santa

Catarina, Brasil.

${ }^{3}$ Instituto Federal de Educação,

Ciências e Tecnologia do Ceará,

Fortaleza, Ceará, Brasil.

${ }^{4}$ Universidade Federal do Rio

Grande do Sul, Porto Alegre, Rio

Grande do Sul, Brasil.

${ }^{5}$ Universidade Regional do Cariri,

Iguatu, Ceará, Brasil.

${ }^{6}$ Universidade Estadual Julio

Mesquita Filho, Presidente Prudente, São Paulo, Brasil.

\section{*Autor correspondente}

Departamento de Educação Física, Universidade Federal do Rio Grande do Sul, Porto Alegre, Rio Grande do Sul, Brasil.

e-mail: glauber_nobre@hotmail.com

Conflito de interesses

Os autores declararam não haver conflito de interesses.

Processo de arbitragem

Recebido: 02/02/2018 Aprovado: 06/06/2018 


\section{Introdução}

Diferentes estudos têm demonstrado o processo de transição nutricional ocorrido no Brasil nos últimos anos. Em menos de duas décadas passou-se de um quadro de diminuição contínua de casos de desnutrição ${ }^{1-5}$ para crescentes prevalências de excesso de peso nas formas de sobrepeso e obesidade $^{2,6}$. A alimentação inadequada tem sido a principal responsável pela ocorrência de distúrbios nutricionais, déficit de crescimento e anemia em crianças em idade escolar ${ }^{7,8}$. A saúde na infância e adolescência vem sendo comprometida pelas diferentes práticas da vida urbana, na qual ocorrem mudanças de comportamento, principalmente referente à alimentação. Tratando-se dos riscos urgentes, aponta-se que os distúrbios nutricionais identificados na infância e na adolescência podem desencadear potenciais fatores de risco predisponentes às doenças cardiovasculares em fases mais avançadas da vida ${ }^{8}$.

As medidas antropométricas de massa corporal e estatura, conforme idade e sexo, são os elementos mais empregados para produção de indicadores de avaliação do estado nutricional ${ }^{9}$. O aumento das prevalências de inadequação nutricionais, especialmente em relação ao excesso de peso, na infância e na adolescência vem originando ampla discussão nos últimos anos e já é estimado como um problema emergente de saúde pública em todo o mundo ${ }^{9-10}$, merecendo especial atenção nos países em desenvolvimento, como o Brasil.

Apesar do reconhecimento desta mudança global nos padrões corporais, no Brasil, há divergências econômicas, culturais e de comportamentos de vida que influenciam diretamente no estado nutricional da população. Entre os estados das regiões Norte e Nordeste, evidencia-se uma dupla carga de problemas associados à inadequação nutricional ${ }^{8,11}$, com tendência a serem mais discrepantes em localidades rurais e povos tradicionais ${ }^{12-13}$.

Programas governamentais têm sido propostos com o intuito de monitorar as condições de saúde e identificar as regiões de maior risco ainda em idades precoces. Entre as políticas públicas nacionais de monitoramento e promoção da saúde da criança e do adolescente no Brasil, destaca-se o Programa Saúde na Escola (PSE), resultado da parceria entre os Ministérios da Saúde e da Educação ${ }^{14}$. O PSE tem sido a principal estratégia inter setorial para promoção de ações contínuas de educação em saúde e avaliação longitudinal das condições de saúde dos escolares ${ }^{14}$. A implementação do PSE nas escolas públicas favorece o acompanhamento do estado nutricional de crianças e adolescentes, além de possibilitar intervençõesde saúde direcionadas aos principais fatores de riscos identificados entre os escolares. Portanto, o presente estudo tem por objetivo investigar possivel défict de estatura para idade de escolares ( 3 a 19 anos) de ambos os sexos de Parintins, AM.

\section{Métodos}

Trata-se de uma pesquisa epidemiológica de delineamento transversal com 1.349 escolares (50,4\% meninos) de 3 e 19 anos, média de idade de 11,3 (desvio padrão $=4,28$ ), provenientes de escolas públicas credenciadas ao Programa Saúde na Escola (PSE) de Parintins, Amazonas. O estudo foi submetido e aprovado pelo Comitê de Ética em Pesquisa em Seres Humanos da Universidade Federal do Amazonas (UFAM) sob o número de parecer 860.883. O diretor de cada escola assinou um termo de consentimento livre e esclarecido (TCLE) autorizando a pesquisa, assim como os responsáveis legais dos alunos.

O município de Parintins situa-se na região do Baixo Amazonas, localizada a leste do Estado do Amazonas. Essa zona equatorial tem um clima quente e úmido e foi povoada por indígenas, principalmente da etnia Sateré-Mawé, e colônias de japoneses que vieram ao Brasil durante a Segunda Guerra Mundial. Segundo dados do Instituto Brasileiro de Geografia e Estatística, a população do município é de aproximadamente 111.575 habitantes $^{15}$.

Segundo fontes documentais da Secretaria Municipal de Saúde, oito escolas estão cadastradas no PSE. A população foi composta por alunos matriculados no ensino infantil, fundamental e médio de seis destas escolas localizadas em regiões equidistantes no município. As escolas elegíveis para participar da pesquisa deveriam, além de estar credenciada ao
PSE, apresentar o cronograma de realização de ações educativas e de avaliação em saúde durante a Semana Saúde na Escola, realizada no mês de junho de 2013.

Um censo escolar foi realizado, sendo incluídos os escolares que atenderam aos seguintes critérios: I) idade entre máxima de 19 anos; II) matriculados em escolas vinculadas ao PSE no ano letivo de 2013; III) com TCLE dos responsáveis legais assinado. Os escolares que não atendiam ao critério de faixa etária foram avaliados, porém os dados não foram considerados nas análises.

A equipe de coleta de dados foi formada por 19 acadêmicos do curso de Educação Física e por um professor coordenador. Nas duas semanas anteriores à pesquisa os acadêmicos foram recrutados e realizam um treinamento com duração média de quatro horas para aplicação e padronização do protocolo de medidas antropométricas e preenchimento da ficha de avaliação física. Nesse período as escolas foram informadas sobre o cronograma de visita e coleta de dados e os pais e/ou responsáveis dos alunos foram informados com antecedência sobre a avaliação e a vestimenta adequada para realização das avaliações antropométricas. As medidas foram realizadas na última semana do mês de junho, com duração de cinco dias, durante o horário e turno letivo dos escolares.

A massa corporal foi aferida com uma balança digital da marca Accumed Balmac, calibrada com precisão de $0,1 \mathrm{~kg}$ e a estatura com uma fita métrica com precisão de $0,1 \mathrm{~cm}$, fixada à parede, em superfície plana de apoio, e com auxílio de um esquadro sobreposto à cabeça dos indivíduos e apoiado à parede, de acordo com procedimentos padronizados16. A partir dessas medidas foi calculado o índice de massa corporal (IMC), dividindo a massa corporal pela estatura elevada ao quadrado.

O desfecho do estudo, estado nutricional, foi avaliado por meio de três indicadores, a massa corporal e estatura adequada para idade e o IMC. Os critérios de classificação adotados foram as curvas de crescimento da Organização Mundial da Saúde ${ }^{17,18}$ As variáveis independentes do estudo foram a idade, calculada a partir da data de nascimento e de avaliação e o sexo (masculino e feminino).

Os dados foram analisados mediante a estatística descritiva (média, desvio padrão, frequência absoluta e relativa). De acordo com o teste de normalidade de Kolmogorov-Smirnov, os dados não apresentaram distribuição normal e as médias em cada grupo etário entre os sexos foram testadas pelo $U$ de Mann-Whitney. Para testar as diferenças entre as proporções dos desfechos entre os sexos em cada grupo etário foi utilizado o teste de Qui-quadrado. As análises foram realizadas no SPSS, versão 15.0 e o nível de significância adotado em todas as análises foi de $5 \%$.

\section{Resultados}

Dos valores antropométricos, a estatura foi a principal variável que apontou diferenças na comparação entre os sexos: nas idades de 4 e dos 15 aos 19 anos os valores foram estatisticamente superiores para meninos e aos 12 anos para as meninas (Tabela 1).

\section{Tabela 1}

Valores descritivos de estatura de crianças e adolescentes do baixo Amazonas de acordo com o sexo e idade (anos). Parintins, AM.

\begin{tabular}{cccccc}
\hline & \multirow{2}{*}{ Idade } & $\mathrm{M}$ & $\mathrm{F}$ & \multicolumn{3}{c}{ Estatura $(\mathrm{m})$} \\
\cline { 2 - 5 } & $\mathrm{n}$ & $\mathrm{n}$ & Média $(\mathrm{DP})$ & Média & \multirow{2}{*}{$\mathrm{p}^{*}$} \\
\hline 3 & 12 & 13 & $0,95(0,03)$ & $0,97(0,04)$ & 0,40 \\
4 & 46 & 53 & $1,00(0,05)$ & $0,97(0,05)$ & $<\mathbf{0 , 0 1}$ \\
5 & 44 & 36 & $1,06(0,04)$ & $1,05(0,05)$ & 0,12 \\
6 & 55 & 51 & $1,12(0,06)$ & $1,11(0,06)$ & 0,21 \\
7 & 14 & 07 & $1,21(0,04)$ & $1,19(0,05)$ & 0,45 \\
8 & 23 & 14 & $1,23(0,04)$ & $1,23(0,07)$ & 0,99 \\
9 & 15 & 22 & $1,30(0,07)$ & $1,30(0,08)$ & 0,95 \\
10 & 39 & 28 & $1,34(0,07)$ & $1,36(0,06)$ & 0,21 \\
11 & 45 & 50 & $1,38(0,06)$ & $1,39(0,09)$ & 0,32 \\
12 & 61 & 78 & $1,40(0,08)$ & $1,44(0,08)$ & $<0,01$ \\
13 & 78 & 91 & $1,48(0,11)$ & $1,49(0,09)$ & 0,27 \\
14 & 68 & 71 & $1,54(0,09)$ & $1,52(0,09)$ & 0,15 \\
15 & 50 & 64 & $1,61(1,00)$ & $1,55(0,08)$ & $<\mathbf{0 , 0 1}$ \\
16 & 52 & 40 & $1,63(0,09)$ & $1,57(0,06)$ & $<\mathbf{0 , 0 1}$ \\
17 & 42 & 26 & $1,63(0,06)$ & $1,55(0,05)$ & $<\mathbf{0 , 0 1}$ \\
18 & 19 & 19 & $1,68(0,07)$ & $1,57(0,07)$ & $<\mathbf{0 , 0 1}$ \\
19 & 17 & 06 & $1,66(0,09)$ & $1,53(0,07)$ & $\mathbf{0 , 0 1}$ \\
\hline
\end{tabular}

Whitney. 
Para a massa corporal foram observadas diferenças nas idades de $4,12,13,17,18$ e 19 anos. Aos 4 anos e dos 17 aos 19 anos, os meninos apresentaram valores superiores às meninas e, aos 12 e 13 anos, as meninas apresentaram maior peso corporal comparado aos meninos (Tabela 2). O IMC apontou diferenças apenas para a idade de 18 anos, com valores superiores para os meninos (Tabela 3 )

Tabela 2

Valores descritivos de massa corporal de crianças e adolescentes do baixo Amazonas de acordo com o sexo e idade (anos), Parintins, AM.

\begin{tabular}{cccccc}
\hline \multirow{2}{*}{ Idade } & \multirow{2}{*}{$\mathrm{M}$} & $\mathrm{F}$ & \multicolumn{3}{c}{ Massa Corporal $(\mathrm{kg})$} \\
\cline { 2 - 5 } & $\mathrm{n}$ & $\mathrm{n}$ & Média $(\mathrm{DP})$ & Média $(\mathrm{DP})$ & \multirow{2}{*}{ p $^{*}$} \\
\hline 3 & 12 & 13 & $14,20(1,64)$ & $14,21(2,77)$ & 0,68 \\
4 & 46 & 53 & $15,73(3,11)$ & $14,40(1,63)$ & $\mathbf{0 , 0 2}$ \\
5 & 44 & 36 & $17,04(1,75)$ & $16,41(1,81)$ & 0,07 \\
6 & 55 & 51 & $19,13(3,04)$ & $18,64(3,00)$ & 0,26 \\
7 & 14 & 07 & $22,29(4,73)$ & $19,62(1,90)$ & 0,09 \\
8 & 23 & 14 & $22,70(2,58)$ & $24,57(6,71)$ & 0,65 \\
9 & 15 & 22 & $26,60(4,85)$ & $26,63(6,87)$ & 0,60 \\
10 & 39 & 28 & $29,47(7,61)$ & $30,08(6,36)$ & 0,71 \\
11 & 45 & 50 & $32,94(7,50)$ & $33,10(6,61)$ & 0,60 \\
12 & 61 & 78 & $34,52(6,00)$ & $37,10(7,24)$ & $\mathbf{0 , 0 3}$ \\
13 & 78 & 91 & $40,26(8,18)$ & $42,01(7,31)$ & $\mathbf{0 , 0 4}$ \\
14 & 68 & 71 & $45,30(9,33)$ & $44,86(7,40)$ & 0,96 \\
15 & 50 & 64 & $50,25(9,34)$ & $47,94(10,18)$ & 0,16 \\
16 & 52 & 40 & $52,67(11,01)$ & $50,02(7,63)$ & 0,31 \\
17 & 42 & 26 & $53,55(8,65)$ & $49,31(7,43)$ & $\mathbf{0 , 0 4}$ \\
18 & 19 & 19 & $58,84(7,99)$ & $47,20(9,05)$ & $<\mathbf{0 , 0 1}$ \\
19 & 17 & 06 & $58,61(12,76)$ & $47,20(6,08)$ & $\mathbf{0 , 0 5}$ \\
\hline M: Masculino; F: Feminino; DP: desvio padrão; ${ }^{*} \mathrm{p}$ valor do teste $\mathrm{U}$ Mann-
\end{tabular}

Whitney.

Tabela 3

Valores descritivos de índice de massa corporal (IMC) de crianças e adolescentes do baixo Amazonas de acordo com o sexo e idade (anos), Parintins, AM.

\begin{tabular}{cccccc}
\hline \multirow{2}{*}{ Idade } & \multirow{2}{*}{$\mathrm{M}$} & $\mathrm{F}$ & \multicolumn{3}{c}{$\mathrm{IMC}\left(\mathrm{kg} / \mathrm{m}^{2}\right)$} \\
\cline { 2 - 5 } & $\mathrm{n}$ & $\mathrm{n}$ & Média $(\mathrm{DP})$ & Méninas & \multirow{2}{*}{ pédia $\left.^{*} \mathrm{DP}\right)$} \\
\hline 3 & 12 & 13 & $15,80(1,36)$ & $15,01(1,78)$ & 0,23 \\
4 & 46 & 53 & $15,55(1,78)$ & $15,24(1,49)$ & 0,69 \\
5 & 44 & 36 & $15,14(1,49)$ & $14,85(1,17)$ & 0,55 \\
6 & 55 & 51 & $15,17(1,54)$ & $15,14(2,01)$ & 0,58 \\
7 & 14 & 07 & $15,42(3,19)$ & $13,86(0,79)$ & 0,08 \\
8 & 23 & 14 & $15,15(1,34)$ & $15,97(2,57)$ & 0,50 \\
9 & 15 & 22 & $15,72(1,75)$ & $15,55(2,77)$ & 0,40 \\
10 & 39 & 28 & $16,32(2,73)$ & $16,32(2,92)$ & 0,79 \\
11 & 45 & 50 & $17,13(2,79)$ & $17,23(3,80)$ & 0,69 \\
12 & 61 & 78 & $17,67(2,57)$ & $17,87(2,76)$ & 0,46 \\
13 & 78 & 91 & $18,44(3,21)$ & $18,99(3,08)$ & 0,14 \\
14 & 68 & 71 & $19,10(3,28)$ & $19,41(3,03)$ & 0,41 \\
15 & 50 & 64 & $19,32(2,59)$ & $20,00(4,16)$ & 0,33 \\
16 & 52 & 40 & $19,79(3,12)$ & $20,40(2,99)$ & 0,19 \\
17 & 42 & 26 & $20,32(3,51)$ & $20,47(2,69)$ & 0,58 \\
18 & 19 & 19 & $20,97(2,78)$ & $19,12(2,90)$ & 0,05 \\
19 & 17 & 06 & $21,22(3,10)$ & $20,27(1,72)$ & 0,62 \\
\hline
\end{tabular}

M: Masculino; F: Feminino; DP: desvio padrão; ${ }^{*} p$ valor do teste U MannWhitney.

As proporções de baixa estatura para as meninas foram significativamente maior para todas as faixas etárias comparadas aos meninos (Figura 1). As proporções gerais dos indicadores foram diferentes entre os sexos somente para a estatura para a idade. Todas as meninas apresentaram estatura baixa $(83,9 \%)$ ou muito baixa $(16,1 \%)$ para a idade e nos meninos o percentual foi de $16,2 \%$ para baixa e $3,4 \%$ para muito baixa $(p<0,01)$. A prevalência de baixo peso para idade foi de $12,5 \%$ para os meninos e de $8,5 \%$ para as meninas e, para a classificação "elevada" a prevalência foi de $3,2 \%$ para os meninos e $2,2 \%$ para as meninas $(p=0,279)$. O baixo peso, segundo o IMC esteve presente em $9,3 \%$ dos meninos e $9,1 \%$ das meninas, enquanto que a proporção do excesso de peso foi de $12,6 \%$ e $11,7 \%$, respectivamente $(p=846)$ (dados não apresentados).

\section{Discussão}

Uma característica do povo de Parintins é a ascendência indígena, embora o presente estudo não tenha realizado investigação das características étnicas dos escolares, dados do IBGE15 revelam que atualmente 512 indígenas declarados Sateré-Mawé residem no ambiente urbano de Parintins/AM, dessas 314 crianças entre 3 e 19 anos.

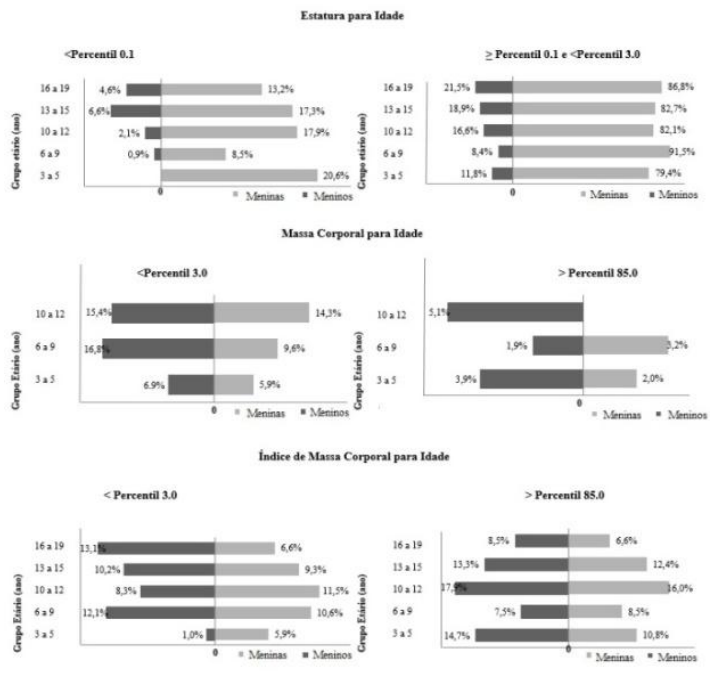

Figura 1. Proporção de inadequação do estado nutricional dos escolares, por sexo, de acordo com as faixas etárias, Parintins, AM. Legenda: * $\mathrm{p}<0,001$

Os principais achados desse estudo revelaram que os meninos são mais altos que as meninas aos 4 e dos 15 aos 19 anos, mais pesados aos 4 e dos 17 aos 19 anos e possuem maior IMC aos 18 anos. Por outro lado, as meninas estavam mais altas que os meninos aos 12 anos e mais pesadas dos 12 aos 13 anos. Em relação às proporções, as meninas apresentaram maiores proporções de déficit em estatura em todos os grupos etários.

Os resultados observados na comparação dos valores médios da estatura entre os sexos em cada idade vão ao encontro da literatura ${ }^{19-20}$ e foram inferiores ao observado em crianças indígenas do Canadá, com idades entre 8 a 14 anos $^{21}$. Pesquisa de abrangência nacional apontou resultados semelhantes quanto à estatura elevada do sexo masculino na faixa etária entre 15 e 17 anos, bem como para as meninas na idade de 12 anos $^{20}$

Em relação à massa corporal para idade, em crianças Xavantes investigadas ${ }^{22}$, as proporções atingiram 16,5\% daquelas com menos de cinco anos e de $9,4 \%$ nas que tinham menos de 10 anos. Entre adolescentes de diferentes regiões do país, foi verificado que as meninas são mais pesadas aos 12 anos e os meninos, ultrapassaram-nas a partir dos 14 anos, contudo esta última diverge dos dados da presente pesquisa, visto que valores superiores de peso para meninos só foram constatados a partir dos 17 anos $^{20}$.

As diferenças encontradas entre os meninos e meninas para os indicadores de estatura e massa corporal para a idade, pode ser explicada pelo estirão de crescimento e maturação, fases do desenvolvimento físico pela qual as meninas passam por volta dos 12 ou 13 anos. Essa fase é caracterizada por aumento no peso corporal e desenvolvimento das características sexuais secundárias, processo que ocorrerá somente por volta de dois anos mais tarde nos meninos ${ }^{19}$.

Todas as meninas apresentaram déficit de estatura e, na comparação das proporções por grupo etário, diferiram dos meninos. Os resultados das pesquisas realizadas na região Norte indicam a nítida presença de desnutrição e denunciam a carente realidade de ações públicas e privadas para mudanças desse perfil ${ }^{8,11,23-24}$. Na capital do Amazonas, Manaus, encontrou-se maior alteração na curva de crescimento, tanto em relação à estatura/idade, quanto para peso/idade, onde 16,1\% das crianças e adolescentes apresentaram déficit acentuado de crescimento $^{25}$. Na faixa etária de 7 a 10 anos, as pesquisas em outros estados da região Norte indicam prevalência de baixo peso para estatura de $11 \%$ em Porto Velho ${ }^{23}$ e $16,6 \%$ no município de Belém ${ }^{24}$. Os resultados supracitados são inferiores as prevalências de baixo peso para estatura desta pesquisa.

Nos dados da Pesquisa Nacional de Demografia e Saúde da Criança e da Mulher, do ano de $2006^{12}$, observou-se déficit de estatura para idade entre as crianças menores de cinco anos, sendo maiores para os meninos comparadoàs meninas, ao contrário do observado neste estudo. A pesquisa destaca ainda, 
o retardo no crescimento das crianças da zona rural do Brasil $(7,6 \%)$ e de forma mais acentuada na região Norte $(14,9 \%)$.

As divergências no aspecto nutricional das crianças e adolescentes, ainda são mais graves quando avaliado residentes da reserva indígena. Investigação realizada com crianças da população indígena Xavante, também identificou que meninos e meninas são mais baixos que as crianças da população-referência. Em crianças com menos de cinco anos, a prevalência de déficit de estatura foi $31,7 \%$ e de $21,6 \%$ naquelas com menos de 10 anos. Quando considerada a adequação da proporção peso/estatura, as prevalências de baixo peso são visivelmente inferiores $(0,9 \%)^{22}$.

A baixa estatura para idade entre crianças menores de cinco anos de aldeias indígenas da etnia Guarani, localizadas na região sudeste, alcançaram as prevalências de $52,4 \%$ para os meninos e $48,4 \%$ para as meninas ${ }^{26}$. Em crianças indígenas $\mathrm{Ti}$ Guarita, dessa mesma faixa etária, a prevalência de baixa estatura foi de $34,7 \%$, com prevalências maiores para os meninos27, e os indígenas Surui menores de 5 anos a prevalência foi de $31,4 \%$ ?

Entre crianças menores de 10 anos de diferentes etnias brasileiras, observou-se prevalências de baixa estatura para idade de 11,1 e $46,3 \%{ }^{13}$, enquanto que em crianças indígenas Pakaanóva, de Rondônia, 45,8\% com idade entre 2 e 10 anos, apresentaram baixos índices de estatura ${ }^{28}$. Entre as crianças indígenas Surui do estado do Amazonas, a prevalência de baixa estatura para idade foi de $25,4 \%^{7}$. As prevalências de baixa estatura encontradas entre os indígenas são superiores a população brasileira, bem como os valores encontrados na população estudada.

O crescimento físico sofre influência da genética, dos mecanismos fisiológicos compensatórios ocasionados pela desnutrição, bem como das condições sociais e ambientais ${ }^{29-30}$. A estatura, especificamente, está condicionada à herança genética dos pais, contudo, os fatores ambientais são os mais influenciadores, principalmente pelas questões sociais em relação às doenças e a nutrição ${ }^{30-31}$

Quando a baixa estatura é de origem genética ela está relacionada ao canal familiar, isto é, as crianças crescem abaixo do percentil 3 e com velocidade de crescimento normal, mas os pais (um ou ambos) possuem estatura baixa. Nesses casos, não há atraso ou prejuízos para o crescimento e essas crianças podem permanecer com baixa estatura na vida adulta. Contudo, quando a origem são condições ambientais, a interferência ocorre no ritmo de crescimento físico final e na estatura ${ }^{25,32}$.

O processo que leva à baixa estatura se dá nos três primeiros anos de vida33, os quais são determinantes, sendo considerado desnutrição crônica se diagnosticado até essa idade, e após ela, ocorre a desnutrição pregressa ${ }^{32}$. No Brasil, uma das causas frequentes de baixa estatura é a desnutrição crônica, independentemente se a manifestação ocorre nos períodos pré ou pós-natal ${ }^{32}$.

Os resultados encontrados nesta pesquisa favorecem a melhoria do cenário de baixo número de publicações sobre o estado nutricional de escolares da região Norte, em especial, do interior do Amazonas. Além disso, tem potencial para contribuir com o avanço científico existente à cerca das condições nutricionais de crianças e adolescentes dessa região, visto que o PSE realiza acompanhamento contínuo da saúde dos escolares. A implementação do PSE em Parintins/AM, prevê a articulação de diversas ações em saúde na escola que devem ocorrer de forma concomitante. Essas ações são apresentadas por componentes ou áreas temáticas agrupadas de acordo com a natureza das ações, como avaliação das condições de saúde dos estudantes, ações de promoção da saúde e prevenção de doenças e agravos, formação de profissionais e jovens para atuarem como multiplicadores, monitoramento da saúde dos estudantes e o monitoramento do próprio programa.

Todavia, algumas limitações devem ser apresentadas, como a escolha das unidades escolares, visto que eram eleitas a participar apenas aquelas vinculadas ao PSE e que fizeram adesão a Semana de Saúde na Escola, o que impossibilita o conhecimento sobre o estado nutricional dos escolares de outras escolas que não são vinculados ao PSE.

\section{Conclusão}

A avaliação do estado nutricional dos escolares do Baixo Amazonas monitorados pelo PSE apontou inadequação para o indicador estatura para a idade em todas as meninas, enquanto que nos meninos a proporção foi de aproximadamente dois em cada 10 avaliados. Espera-se, a partir dos resultados encontrados, consolidar e ampliar a adesão das escolas a atividades de monitoramento de saúde do PSE, bem como medidas de prevenção contra a desnutrição infantil, déficits de crescimento e excesso de peso, as quais sendo iniciadas logo na idade escolar e mantidas até a adolescência, têm resultados mais eficazes. Além disso, possibilitar futuras pesquisas, que objetivem traçar tendências de crescimento e estado nutricional desta população, mediante a manutenção das ações do PSE e da articulação entre a gestão municipal e a universidade.

\section{Referências}

1. Chaves VLV, Freese E, Lapa MT, Cesse EAP, Vasconcelos ALR Evolução espaço temporal do sobrepeso e da obesidade em adolescentes masculinos brasileiros, 1980 a 2005. Cad. Saúde Pública 2010 26(7):1303-13

2. Coutinho JG, Gentil CP, Toral N. A desnutrição e obesidade no Brasil: o enfrentamento com base na agenda única da nutrição. Cad Saúde Pública 2008; 24(Sup.2):S332-S340.

3. Filho MB, Rissin A. A transição nutricional no Brasil: tendências regionais e temporais. Cad. Saúde Pública 2006; 19(Sup. 1):S181-S191.

4. Tardido AP, Falcão MC. O impacto da modernização na transição nutricional e obesidade. Rev Bras Nutr Clin 2006; 21(2):117-24.

5. Monteiro CA, Benicio MHD, Konno SC, Silva ACF, Lima ALL, Conde WL. Causas do declínio da desnutrição infantil no Brasil, 1996-2007. Rev Saúde Púb 2009; 43(1):35-43.

6. Pimenta TAM, Rocha R, Marcondes NAV. Políticas Públicas de Intervenção na Obesidade Infantil no Brasil: uma Breve Análise da Política Nacional de Alimentação e Nutrição e Política Nacional de Promoção da Saúde. Cient Ciênc Biol Saúde UNOPAR 2015; 17(2):139-46.

7. Orellana JDY, Coimbra Jr. CEA, Lourenço AEP, Santos RV. Estado Nutricional e anemia em crianças Suruí, Amazônia, Brasil. J Pediatr 2006; 82(5): 383-388.

8. Reis CEG, Vasconcelos IAL, Barros JFN. Políticas públicas de nutrição para o controle da obesidade infantil. Rev paul pediatr 2011; 29(4):625-33.

9. World Health Organization/WHO. Obesity: preventing and managing the global epidemic. Geneva: World Health Organization. 2000.

10. Wang $\mathrm{Y}$, Lobstein $\mathrm{T}$. Worldwide trends in childhood overweight and obesity. Int J Pediatr Obes 2006; 1(1):11-25.

11. Maia MMM. Estado nutricional, consumo alimentar, deficiência de micronutrientes e doenças parasitárias em crianças de 0 a 10 anos de idade atendidas em serviços de saúde da cidade de Manaus, Amazonas. [Dissertação de Mestrado -Programa de Pós Graduação em Parasitologia]. Belo Horizonte (MG): Universidade Federal de Minas Gerais, 2006.

12. Lima ALL, Monteiro CA, Konno SC, Conde WL. Avaliação antropométrica do estado nutricional de crianças e mulheres em idade fértil. In: Ministério da Saúde. Relatório Final: Pesquisa Nacional de Demografia e Saúde da Mulher e da Criança; 2008. p.252-262. disponível em:http://bvsms.saude.gov.br/bvs/pnds/img/relatorio_final_pnds2006.pdf [2015out 21]

13. Licio JSA. Estado nutricional de crianças indígenas no Brasil: uma revisão sistemática da literatura científica. [Dissertação de Mestrado - Ciências na área da Saúde Pública]. Rio de Janeiro (RJ): Fundação Oswaldo Cruz, 2009.

14. Brasil. Decreto Federal no 6.286, de 05 de dezembro de 2007. Dispõe sobre o Programa Saúde na Escola (PSE) e dá outras providências. Brasília: Casa Civil, 2007.

15. Instituto Brasileiro de Geografia e Estatística. IBGE Cidades. 2015 Disponível

http://www.cidades.ibge.gov.br/xtras/perfil.php?lang=\&codmun=130340\&s earch=amazonas $\mid$ parintins

16. Alvarez BR, Pavan AL. Alturas e comprimentos. In: Petroski EL (ed.). Antropometria: técnicas e padronizações. 5.ed. Várzea Paulista: Fontoura, 2011, p.31-44

17. World Health Organization. Child Growth Standards based on length/height, weight and age. Acta Paediatr 2006; 450(Suppl.):76-85.

18. Onis $\mathrm{M}$ et al. Development of a WHO growth reference for school-aged children and adolescents. Bull World Health Organ 2007; (85):660-7.

19. Malina RM, Bouchard C, Bar-Or O. Crescimento, maturação e atividade física. 2nd ed. São Paulo: Phorte; 2009. p. 466-467.

20. Silva DAS, Pelegrini A, Petroski EL, Gaya ACA. Comparação do crescimento de crianças e adolescentes brasileiros com curvas de referência para crescimento físico: dados do Projeto Esporte Brasil. Rev Soc Bol Ped 2012; 51(3):211-17.

21. Medehouenou TCM, Ayotte P, St-Jean A, Meziou S, Roy C, Muckle G, et al. Overweight and Obesity Prevalence Among School-Aged Nunavik Inuit Children According to Three Body Mass Index Classification Systems. Journal of Adolescent Health 2015; 57:31-36.

22. Leite MS, Santos RV, Gugelmin SA, Coimbra Jr. CEA. Crescimento físico e perfil nutricional da população indígena Xavánte de Sangradouro-Volta Grande, Mato Grosso, Brasil. Cad. Saúde Pública 2006; 22(2): 265-276.

23. Farias ES, Guerra-Júnior G, Petroski EL. Estado Nutricional de escolares em Porto Velho, Rondônia. Rev.Nutr. 2008; 21(4):401-409.

24. Neves OMD, Brasil ALD, Brasil LMBF, Taddei JAAC. Antropometria de escolares ao ingresso no ensino fundamental na cidade de Belém, Pará, 2001. Rev. Bras. Saúde Matern. Infant. 2006; 6(1):39-46.

25. Ramalho MHS, Santos JOL, Soares AA, Machado Z, Maria WB, Nazario PF, Nobre GC. Physical growth in children and adolescents of three Brazilian cities. J Health Sci Inst 2011; 29(4):276-9. 
26. Barreto CTG, Cardoso AM, Coimbra Jr CEA. Estado nutricional de crianças indígenas Guarani nos estados do Rio de Janeiro e São Paulo, Brasil. Cad. Saúde Pública 2014; 30(3):657-662.

27. Menegolla IA, Drachler ML, Rodrigues IH, Schwingel LR, Scapinello E, Pedroso MB, Leite JCC. Estado nutricional e fatores associados à estatura de crianças da Terra Indígena Guarita, Sul do Brasil. Cad. Saúde Pública 2006; 22(2):395-406.

28. Escobar AL, Santos RV, Coimbra J. Avaliação Nutricional de Crianças Indígenas Pakaanóva (Wari'), Rondônia, Brasil. Rev Bras Saúde Matern Infant 2003; 3(4):457-461.
29. Tanner JM. A History of the Study of Human Growth. London: Cambridge University Press; 1981

30. Vitolo MR, Campagnolo PDB, Gama CM. Factors associated with risk of low dietary fiber intake in adolescents. J Pediatr 2007; 83(1): 47-52.

31. Guedes DP. Crescimento e desenvolvimento aplicado à Educação Física e ao Esporte. Rev. bras. Educ. Fís. Esporte 2011; 25: 127-40.

32. Zeferino AMB, Barros Filho AA, Bettiol H, Barbieri MA. Acompanhamento do crescimento. J Pediatr 2003; 79(Suppl.1):S23-S32.

33. Habicht JP, Martorell R, Yarbrough C, Malina RM, Klein RE. Height and weight standards for preschool children. How relevant are ethnic differences in growth potential? Lancet 19741:611-5. 